\title{
"Cytochrome B"- Analysis of Hydrophobicity, Surface Accessibility, Antigenicity and Prediction of MHC I and MHC II Binders from Dracunculiasis
}

Sonu Mishra and Virendra S Gomase*

Department of Biotechnology, Mewar University, Chittorgarh, India

\begin{abstract}
In this study, Cytochrome $b$ (mitochondrion) protein has been used to investigate its role in antigenicity. Cytochrome b (mitochondrion) protein sequences (367 aa protein) is analyzed through different types B- cell epitope prediction methods. We found that the region of maximal hydrophilicity is likely to be an antigenic site, having hydrophobic characteristics, because the terminal regions of antigen protein is solvent accessible and unstructured, antibodies against those regions are also likely to recognize the native protein. It was seen that an antigen protein is hydrophobic in nature and contains segments of low complexity and high-predicted flexibility. The predicted antigenic protein segments of Cytochrome b (mitochondrion) can take active part in the host immune reactions. In this research, we have also used PSSM and SVM algorithms for the prediction of MHC class I and II binding peptide, antigenicity, Solvent accessibility, polar and nonpolar residue to analyse the regions that are likely exposed on the surface of proteins which are potentially antigenic that allows potential drug targets to identify active sites against infection as well as to design effective drug for treatment.
\end{abstract}

Keywords: Dracunculus medinensis; Dracunculiasis; Epitope; Antigen; Protein; Cytochrome b (mitochondrion)

\begin{abstract}
Abbreviations
MHC I: Major Histocompatibility Complex-Class I; MHC I: Major Histocompatibility Complex-Class II; PSSM: Position Specific Scoring Matrices; SVM: Support Vector Machine; GWD: Guinea worm disease; UniProt: The Universal Protein Resource; NCBI: National Center for Biotechnology Information; TAP: Transporter Associated with Antigen Processing; HPLC: High Performance Liquid Chromatography; TapPred: TAPPred is an on-line service for predicting binding affinity of peptides toward the TAP transporter. The prediction of TAP binding peptides is important in order to identify the MHC class-1 restricted T cell epitopes. The Prediction is based on cascade SVM, using sequence and properties of the amino acids. The correlation coefficient of 0.88 was obtained by using jack-knife validation test
\end{abstract}

Rankpep: This server predicts peptide binders to MHCI and MHCII molecules from protein sequence/s or sequence alignments using Position Specific Scoring Matrices (PSSMs). In addition, it predicts those MHCI ligands whose C-terminal end is likely to be the result of proteasomal cleavage.

\section{Introduction}

Dracunculiasis, is caused by a $60-100 \mathrm{~cm}$ long nematode worm, Dracunculus medinensis, via a drinking of contaminated water infected with copepod Cyclops (intermediate host). Dracunculiasis has been known to humankind since antiquity. Guinea worm the largest tissue parasite with unusual life cycle with incubation period of the approximately more than a year with six developmental stages. This one of the most neglected tropic parasite which bears clinical importance and needs to be eradicated after small pox [1]. Mature and adult female after the copulation produces millions of eggs in its uterus, and is predominantly localized in the lower extremities (80-90\%). After an incubation period the female worm release the larvae which induces a painful blister (1 to $6 \mathrm{~cm}$ diameter) on the skin of lower limbs (Figure 1a) [2]; the person develop a slight fever, local skin redness, swelling and severe pruritus around the blister. Other symptoms include diarrhoea, nausea, vomiting and dizziness. The severity of the wound infections in the infected individual led to a more complications such as redness and swelling of the skin (cellulitis), boils (abscesses), generalized infection (sepsis), joint infections (septic arthritis) that can cause the joints to lock and deform (contractures), lock jaw (tetanus). The blister burst within 1 to 3 days and female worms one or more slowly comes out from the wounds (Figure 1b) which causes an excoriating burning sensation and pain $[3,4]$. Immersing or pouring water over the blister provides pain relief. But this the moment that adult female is exposed to the external environment [5]. During emergence of the limbs in open water sources it recognizes the temperature difference and releases the milky white liquid in the water which contains millions of immature larvae, when larvae released in water are ingested by copepods where they mount twice and become infective larvae within two weeks [6].

The D. medinensis antigen peptides can be most desirable segment for the subunit vaccine development because with the single epitope, the immune response can be generated in large population. This approach is usually based on the phenomenon of cross-protection, whereby infected with the mild strain and is protected against a more severe strain of the same. The phenotype of the resistant transgenic hosts includes fewer centers of initial infection, a delay in symptom development and low accumulation. The World Health Assembly was called in the 1986 in order to eliminate dracunculiasis completely. The global Guinea Worm Eradication Program, supported by The Carter Center, World Health Organization (WHO), UNICEF, CDC, and other partners, began assisting ministries of health of countries in which Dracunculiasis is endemic in meeting this goal. At that time, an estimated 3.5 million cases occurred each year in 20 countries in Africa and Asia. Dracunculiasis remains endemic in four countries

*Corresponding author: Virendra S. Gomase, Department of Biotechnology, Mewar University, Chittorgarh, India, Tel: +919423467927; E-mail: gomase.viren@ gmail.com

Received December 09, 2015; Accepted January 15, 2016; Published January 19, 2016

Citation: Mishra S, Gomase VS (2016) "Cytochrome B"-Analysis of Hydrophobicity, Surface Accessibility, Antigenicity and Prediction of $\mathrm{MHC}$ I and $\mathrm{MHC}$ II Binders from Dracunculiasis. Med chem 6: 041-046. doi:10.4172/2161-0444.1000322

Copyright: $\odot 2016$ Mishra S, et al. This is an open-access article distributed under the terms of the Creative Commons Attribution License, which permits unrestricted use, distribution, and reproduction in any medium, provided the original author and source are credited. 


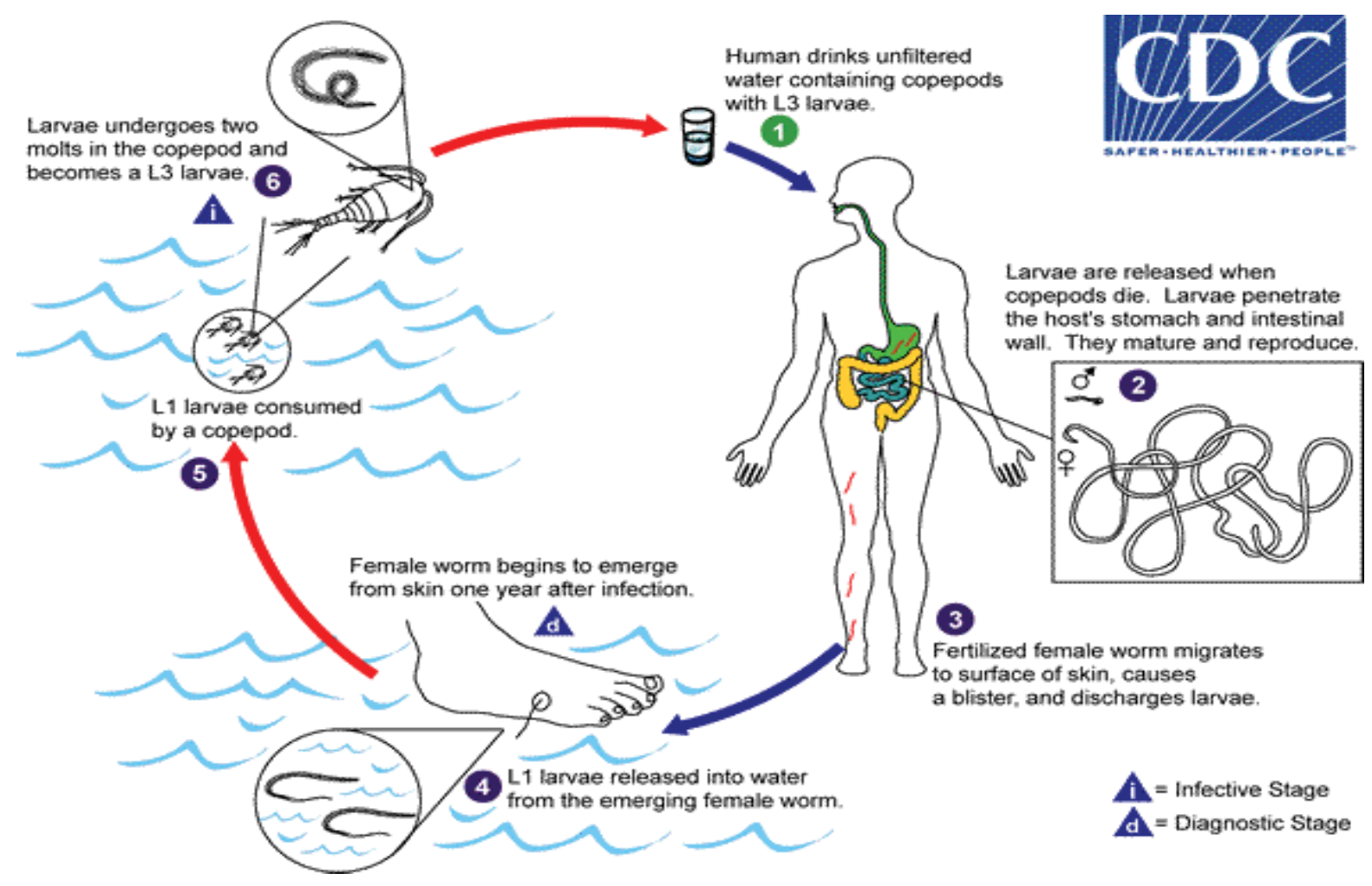

Figure 1: Guinea worm life cycle and interventions to interrupt transmission (Source: www.cdc.gov/parasites/guineaworm/biology.html).

in 2014 (South Sudan, Chad, Mali, and Ethiopia), but the number of overall reported incidence is decreases in 2013 by $73 \%$ and in 2014 by $71 \%$ compared with 2012. Failures in surveillance and containment, lack of clean drinking water, insecurity in Mali and parts of South Sudan, and an unusual epidemiologic pattern in Chad are the main remaining challenges to dracunculiasis eradication [7,8]. A case of Onchocerca volvulus has been reported in the Cameroon which is mimicking Dracunculus medinensis [9]. More than two decades after the International Drinking Water Supply and Sanitation Decade (IDWSSD) implemented by the United Nations (1981-1990) [10], the disease still lingers, underscoring the daunting challenge of disease control, as has been the case of the failure of previous attempts to eradicate diseases like malaria, hookworm and yaws [11]. Till date there is no accurate and efficient curative drug or vaccine is available against dracunculiasis [12]. The investigation suggests that the immunity is not developed by the infected individual $[13,14]$. Study suggest that, the Dracunculus medinensis -specific antibodies (total, IgG1 and IgG4) during the time of patency, which were significantly higher than the levels measured in the same individuals eight months later, except for a few individuals who had developed a new patent infection [15]. Whereas, variation in the IgE value is relatively negotiable and constant before, during the infection and after the recovery. There is possibility that variation in antibody production is regulated by infected larvae (i.e., by transmission) and/or by adult worms (i.e., by patency) is still need to be clear out. It is possibility that increased production of IgG1, IgG4 during the time of patency plays a role in blocking or protecting immune responses otherwise it could have killed ingested infected larvae [16]. In this study Cytochrome b (mitochondrion) has been used to investigate its role in antigenicity and hydrophobicity. Cytochrome $\mathrm{b}$ protein present in the mitochondria of eukaryotic cells. It acts as an important part of the electron transport chain and is the main subunit of transmembrane cytochrome bcl and b6f complexes. Cytochrome b/ b6 isan integral membrane protein consists of the approximately 400 amino acid residue which probably has 8 transmembrane segments. Cytochrome b/b6 non-covalently binds to b562 and b566 heme groups. The four conserved histidine residue in the ligands of the iron atoms of these two heme groups has been postulated $[17,18]$. The cytochrome $\mathrm{b}$ is the central redox catalytic subunit of the quinol: cytochrome $\mathrm{c} /$ plastocyanin oxidoreductases. The cytochrome $b$ functionally involves in the binding of the quinine substrate and actively responsible for the transmembrane electron transfer through which the protonmotive force is created/generated from redox energy. Cytochrome b also carries the binding domain of the various inhibitors and quinine antagonists bindind sites, which subsequently inhibits the oxidoreductase. Esposti et al. able to identify the amino acid residues in cytochrome $b$ which has the probability to be involved in the binding of the inhibitors and, by extrapolation, quinone/quinol [18]. Cytochrome b is also known as the $\mathrm{bcl}$ complex or ubiquinol-cytochrome $\mathrm{c}$ reductase, which is the part of the respiratory chain complex III. The cytochrome b6/ b6f complex is the analogous protein present in the plant chloroplasts and cyanobacteria, which is a part of the plastoquinone-plastocyanin reductase. These complexes are involved in pumping of protons in order to create a PMF, electron transport. The proton gradient is finally promote the ATP generation. These complexes play a vital role in cells [19]. The intolerance in human patients is due to the result of the mutation in cytochrome; though very few severe multi-system pathologies have also been reported [20]. In Plasmodium falciparum and P. berghei a singlepoint mutations in cytochrome $b$ is associated with resistance to the anti-malarial drug atovaquone [21]. It has also reported that mutations in the MT-CYB (mitochondrial cytochrome b) geneis responsible for the deficiency of mitochondrial complex III. This mutation in the gene is the cause of the condition which characterized by muscle weakness (myopathy) and pain especially during exercise (i.e., exercise intolerance). The severity due to this mutation in affected individual 
can suffer from the problems like liver, kidneys, heart, and brain. These tissues required a huge amount of the energy for its functionality, researcher believes that impaired oxidative phosphorylation can lead to cell death which inturns causes the deficiency of the various features of the mitochondrial complex. The accurate position of the MT-CYB gene in mitochondrial DNA (mtDNA) can reveal and unfold the underlying the reason that only few people suffer from more severe features of the condition than others. The percentage of mutated mitochondrial DNA is highest in the skeletal muscles in most of people with MT-CYBrelated mitochondrial complex III deficiency, which further interprets the finding of myopathy in these individuals. The mutation is most prevalent in muscle tissue is still imprecise. Antigenicity prediction of the protein from $D$. medinensis can play an important role in prototype synthetic vaccine development and as well as for target validation.

\section{Methodology}

B-cell epitopes are the sites of molecules that are recognized by antibodies of the immune system. Knowledge of B-cell epitopes may be used in the design of vaccines and diagnostics tests. It is therefore of interest to develop improved methods for predicting B-cell epitopes [22]. In this research work, antigenic epitopes of antigen protein Cytochrome $\mathrm{b}$ (mitochondrion) from $D$. medinensis is determined using the Gomase in 2007, Bepipred Linear Epitope Prediction, Emini Surface Accessibility Prediction, Karplus and Schulz Flexibility Prediction, Kolaskar and Tongaonkar Antigenicity, Parker Hydrophilicity Prediction [23-29].

\section{Database searching}

The antigenic protein sequence of Cytochrome $b$ from Dracunculus medinensis was retrieved from www.ncbi.nlm.nih.gov, UniProt databases are initially the most important [30-32].

\section{Prediction of antigenicity}

Prediction of antigenicity program predicts those segments from antigen cytochrome $b$ protein that are likely to be antigenic by eliciting an antibody response. In this research work antigenic epitopes of Dracunculus medinensis antigen Cytochrome b (mitochondrion) are determined by using the Hopp and Woods, Welling, Parker, Bepipred, Kolaskar and Tongaonkar antigenicity methods [33-37].

\section{Prediction of MHC binding peptide}

The major histocompatibility complex (MHC) peptide binding of Dracunculus medinensis is predicted using neural networks trained on $\mathrm{C}$ terminals of known epitopes. Rankpep predicts peptide binders to MHC-I ligands whose C-terminal end is likely to be the result of proteosomal cleavage using Position Specific Scoring Matrices (PSSMs). Support Vector Machine (SVM) based method for prediction of promiscuous MHC class II binding peptides from protein sequence; SVM has been trained on the binary input of single amino acid sequence [38-41].

\section{Prediction of antigenic peptides by cascade SVM based TAP- Pred method}

In the present study, we predict cascade SVM based several TAP binders which was based on the sequence and the features of amino acids [42]. We found the MHCI binding regions, the binding affinity of Cytochrome b (mitochondrion).

\section{Solvent accessible regions}

We also analyzed the solvent accessible regions of proteins having highest probability that a given protein region lies on the surface of a protein Surface Accessibility, backbone or chain flexibility by Emini et al. [43] and Karplus and Schulz [44]. By using different scale we predict the hydrophobic and hydrophilic characteristics of amino acids that are rich in charged and polar residues [45-53].

\section{Results and Interpretations}

The Dracunculus medinensis antigen Cytochrome b (mitochondrion), contain a long residue of 367 amino acids with 359 nonamers.

MEVLFKYLNSMVVVLPSSKSLDLNWNYGSMLGMILFFQIFTGFLLSFFYSNDSLLAFDSVQYIMYDVNYGWLYRVFHFN GASLFFVFLYLHFFKGLFNSSYRLSLVWLSGVFIIFFVILEAFMGYVLVWAQMSFWACVVITSLLSVVPFFGGDLVLWVWGGFTVSGATLKFFFAVHFLLPWFIFVLVVFHLIMLHVTGSTSVLSGFGDYDKIGFFPYYWLKDSYNLVVWLFFFVFVFSCPFVLGDPEMFLLANCLMSPVHIVPEWYFLFAYAILRAFPNKFLGVVMLVFSIFVFVIFVFINSYVSVFDVLVDFFVWFFLFVVLLLSWLGQCPVEWPYVFFSVFYSLVYFILIFMVFFQGLFFKLLYF

\section{Prediction of antigenic peptides}

In this study, we found the antigenic determinants by finding the area of greatest local hydrophilicity. The Hopp-Woods scale Hydrophilicity Prediction Result Data found high in position: 20 (Max score: 0.389 ) in a protein, assuming that the antigenic determinants would be exposed on the surface of the protein and thus would be located in hydrophilic regions (Figure 2). Welling antigenicity plot gives value as the log of the quotient between percentage in a sample of known antigenic regions and percentage in average proteins and prediction result data found high in position: 19 with high score: 0.406 . We also study Hydrophobicity plot of HPLC / Parker Hydrophilicity prediction result data found i.e., the maximum predicted residues at the position 19 (Residue: $\mathrm{K}$ ) is 16-PSSKSLD-22 and the maximum score: 4.014, BepiPred predicts the location of linear B-cell epitopes Result found at position 20 (Residue: S) with Max Score: 0.520, Kolaskar and Tongaonkar antigenicity methods. Predicted peptides result found are

4 - L F K Y L N S M V V V L P S S K S - 20,31 - L G M I L F F Q I F T G F L L S F F Y S N D S L L A F D S V Q Y I M Y D V N Y 69, 71 - W L Y R V F H F N G A S L F F V F L Y L H F F K G - 95, 97 FNSSYRLSLVWLSGVFIIFFVILEAFMGYVLVWA-130,133SFWACVVITSLLSVVPFFGGDLVLWV-158,160-GGFTVSGAT LKFFFAVHFLLPWFIFVLVVFHLIMLHVTGSTSVLSG-205,211KIGFFPYYWL-220,222-DSYNLVVWLFFFVFVFSCPFVLG-244,247EMFLLANCLMSPVHIVPEWYFLFAYAILRA-276,278-PNKFLGVV MLVFSIFVFVIFVFINSYVSVFDVLVDFFVWFFLFVVLLLSWLGQC PVEWPYVFFSVFYSLVYFILIFMVFFQGLFF-362 and the predicted antigenic fragments can bind to MHC molecule is the first bottlenecks in vaccine design.

\section{Solvent accessible regions}

We also predict solvent accessible regions in proteins; different measurement was performed for the prediction of antigenic activity, surface region of peptides. Emini et al. predicts the highest probability i.e., found in position 222 (Residue: D) i.e., 220-LKDSYN-225, and in the position 223 (Residue: S) i.e., 221-KDSYNL-226, that a given protein region lies on the surface of a protein and are used to identify antigenic determinants on the surface of proteins, Karplus and Schulz. High score is found i.e., found highest score: 1.103 at position 18 (Residue: S) i.e., 15-LPSSKSL -21. Predict backbone or chain flexibility on the basis of the known temperature B factors of the a-carbons. The hydrophobicity and hydrophilic characteristics of amino acids is determined by using different scales that are rich in charged and polar residues i.e., Sweet 

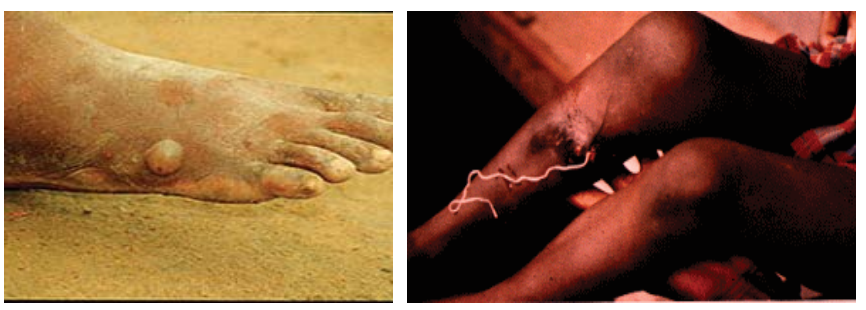

Figure 2: (a) A Blister (1-6 cm diameter) on the skin of lower limbs. (b) The worm coming out after blister burst.

et al. hydrophobicity prediction Result Data found high in position: 353, Max score: 1.481, Kyte and Doolittle result high in position: 115 , Max score: 3.789, Abraham and Leo result high in position: 233, Max score: 2.353, Bull and Breese result high in position: 164, Max score: 0.197 , Miyazawa result high in position: 353 , Max score: 8.759 , Guy result high in position: 20 , Max score: 0.288 , Wolfenden result high in position: 321 , Max score: 1.202 , Roseman result high in position: 233 , Max score: 1.989, Wilson position: 233, Max score: 7.089, Cowan in position: 115, Max score: 1.667, Chothia in position: 115, Max score: 0.537 .

\section{Prediction of MHC binding peptide}

We found the binding of peptides to a number of different alleles using Position Specific Scoring Matrix. Cytochrome b (mitochondrion) of Dracunculus medinensis antigen, with sequence 367 amino acid residues long, having 359 nonamers. MHC molecules are cell surface proteins, which actively participate in host immune reactions and involvement of MHC-I and MHC-II in response to almost all antigens. We have predicted MHC-I peptide binders of Cytochrome b (mitochondrion) from Dracunculus medinensis and was tested with on a set of 4 different alleles i.e., $\mathrm{H} 2-\mathrm{Db}$ (mouse) 8 mer, $\mathrm{H} 2-\mathrm{Db}$ (mouse) 9 mer, $\mathrm{H} 2-\mathrm{Db}$ (mouse) 10mer and H2-Db (mouse) 11 mer and MHCII peptide binders for I_Ab.p, I_Ad.p, I_Ag7.p alleles highlighted in red represent predicted binders. Here RANKPEP report PSSM-specific binding threshold and is obtained by scoring all the antigenic peptide sequences included in the alignment from which a profile is derived, and is defined as the score value that includes $85 \%$ of the peptides within the set. Peptides whose score is above the binding threshold will appear highlighted in red and peptides produced by the cleavage prediction model are highlighted in violet. We also use a cascade SVM based TAPPred method which found 80 High affinity TAP Transporter peptide regions which represents predicted TAP binders residues which occur at N and C termini from Dracunculus medinensis (Cytochrome $\mathrm{b}$ (mitochondrion)).

\section{Discussion}

In this study, we found the antigenic determinants by finding the area of greatest local hydrophilicity. Hopp and Woods hydrophobicity scale is used to identify of potentially antigenic sites in proteins by analyzing amino acid sequences in order to find the point of greatest hydrophilic. Hydrophilicity Prediction result data found high in sequence in position: 20 (Max score: 0.389 ) in a proteinthis scale is basically a hydrophilic index where apolar residues have been assigned negative values. The Window size of 5-7 is good for finding hydrophilic regions, greater than 0 values are consider as hydrophilic which is consider as antigenic. Welling et al. used information on the relative occurrence of amino acids in antigenic regions to make a scale which is useful for prediction of antigenic regions and the predicted result data found high in sequence in position: 19 with high score: 0.406 . Welling et al. antigenicity plot gives value as the log of the quotient between percentage in asample of known antigenic regions and percentage in average proteins. We also study Hydrophobicity plot of HPLC/ Parker Hydrophilicity Prediction Result Data found i.e., the maximum predicted residues at the position 19 (Residue: K) is 16-PSSKSLD-22 and the maximum score: 4.014 . BepiPred predicts the location of linear B-cell epitopes Result found at position 20 (Residue: S) with Max Score: 0.520 . There are 10 antigenic determinant sequences is found by Kolaskar and Tongaonkar antigenicity scales the results show highest pick at

4 - L F K Y L N S M V V V L P S S K S - 20,3 1 - L G M I L F F Q I F T G F L L S F F Y S N D L L A F D S V Q Y I M Y D V N Y - 69, 71 W L Y R V F H F N G A S L F F V F L Y L H F F K G - 95, 97 - F N S S Y RLSLVW L S G V F I I F F V ILE A F M G Y V L V W A - 130,133 SFWACVVITSLLSVVPFFGGDLVLWV-158,160-GGFTVSGAT LKFFFAVHFLLPWFIFVLVVFHLIMLHVTGSTSVLSG-205,211KIGFFPYYWL-220,222-DSYNLVVWLFFFVFVFSCPFVLG-244,247EMFLLANCLMSPVHIVPEWYFLFAYAILRA-276,278-PNKFLGVV MLVFSIFVFVIFVFINSYVSVFDVLVDFFVWFFLFVVLLLSWLGQC PVEWPYVFFSVFYSLVYFILIFMVFFQGLFF-362.

Result of determined antigenic sites on proteins has revealed that the hydrophobic residues if they occur on the surface of a protein are more likely to be a part of antigenic sites. This method can predict antigenic determinants with about $75 \%$ accuracy and also gives the information of surface accessibility and flexibility. Further this region form beta sheet which show high antigenic response than helical region of this peptide and shows highly antigenicity. X-Ray Diffraction with Resolution $1.49 \AA$ 3D Structure of the Dracunculus medinensis antigen-cytochrome $\mathrm{b}$ is predicted by $\mathrm{PDB}$ vive. We generate a purified protein for analysis of the chosen target and then structure determined the target experimentally to evaluate their similarity to known protein structures and to determine possible relationships that are identifiable from protein sequence alone. The target structure will also serve as a detailed model for determining the structure of peptide within that protein structure. We predict Solvent accessibility by using Emini et al. the result found the highest probability i.e., in position 222 (Residue: D) i.e., 220-LKDSYN-225, and in the position 223 (Residue: S) i.e., 221-KDSYNL-226, that a given protein region lies on the surface of a protein and are used to identify antigenic determinants on the surface of proteins. This algorithm also used to identify the antigenic determinants on the surface of proteins and Karplus and Schulz predict backbone or chain flexibility on the basis of the known temperature B factors of the a-carbons here we found the result with High score found i.e., found highest score: 1.103 at position 18 (Residue: S) i.e., 15-LPSSKSL -21. We predict Solvent accessibility of Dracunculus medinensis antigen Cytochrome $\mathrm{b}$ for delineating hydrophobic and hydrophilic characteristics of amino acids. Solvent accessibility used to identify active site of functionally important residues in membrane proteins. Solvent-accessible surface areas and backbone angles are continuously varying because proteins can move freely in a threedimensional space. The mobility of protein segments which are located on the surface of a protein due to an entropic energy potential and which seem to correlate well with known antigenic determinants. We also found the Sweet et al. hydrophobicity prediction result data found high in position: 353, Max score: 1.481, Kyte and Doolittle result high in position: 115, Max score: 3.789, Abraham and Leo result high in position: 233, Max score: 2.353, Bull and Breese result high in position: 164, Max score: 0.197 , Miyazawa result high in position: 353 , Max score: 8.759 , Guy result high in position: 20, Max score: 0.288 , Wolfenden result high in position: 321 , Max score: 1.202, Roseman result high in position: 233, Max score: 1.989, Wilson et position: 233, 
Max score: 7.089, Cowan in position: 115, Max score: 1.667, Chothia in position: 115 , Max score: 0.537 . These scales are a hydrophilic with a polar residues assigned negative value. Because the $\mathrm{N}$ - and $\mathrm{C}$ - terminal regions of proteins are usually solvent accessible and unstructured, antibodies against those regions recognize the antigenic protein. In this study, we found predicted MHC-I peptide binders of protein for 8mer_H2_Db alleles with the consensus sequence QNWNCCTI that yields the maximum score i.e., 52.494, 9mer_H2_Db with, the consensus sequence FCIHNCDYM that yields the maximum score i.e., $50.365,10$ mer_H2_Db with, the consensus sequence SGYYNFFWCL that yields the maximum score i.e., 58.858, 11mer_H2_Db with, the consensus sequence CGVYNFYYCCY that yields the maximum score i.e., 79.495 and I_Abwith the consensus sequence YYAPWCNNA that yields the maximum score i.e., 35.632, I_Ad with the consensus sequence QMVHAAHAE that yields the maximum score i.e., 53.145, MHC-II I_Ag7 with the consensus sequence WYAHAFKYV that yields the maximum score i.e., 40.873 for MHC II allele was tasted. We also use a cascade SVM based TAPPred method which found 160 High affinity TAP Transporter peptide regions which represents predicted TAP binders residues which occur at $\mathrm{N}$ and $\mathrm{C}$ termini from Dracunculus medinensis antigen Cytochrome b. TAP is an important transporter that transports antigenic peptides from cytosol to ER. TAP binds and translocate selective antigenic peptides for binding to specific MHC molecules. The efficiency of TAP-mediated translocation of antigenic peptides is directly proportional to its TAP binding affinity. Thus, by understanding the nature of peptides, that bind to TAP with high affinity, is important steps in endogenous antigen processing. The correlation coefficient of 0.88 was obtained by using jackknife validation test. In this test, we found the MHCI and MHCII binding regions. T cell immune responses are derived by antigenic epitopes hence their identification is important for design synthetic peptide vaccine. T cell epitopes are recognized by MHCI molecules producing a strong defensive immune response against antigenic protein Cytochrome b. Therefore, the prediction of peptide binding to MHCI molecules by appropriate processing of antigen peptides occurs by their binding to the relevant MHC molecules. Because, the C-terminus of MHCI-restricted epitopes results from cleavage by the proteasome and thus, proteasome specifity is important for determing T-cell epitopes. Consequently, RANKPEP also focus on the prediction of conserved epitopes. C-terminus of MHCI-restricted peptides is generated by the proteasome, and thus RANKPEP also determines whether the C-terminus of the predicted MHCI-peptide binders is the result of proteasomal cleavage. Moreover, these sequences are highlighted in purple in the output results. Proteasomal cleavage predictions are carried out using three optional models obtained applying statistical language models to a set of known epitopes restricted by human MHCI molecules as indicated here.

\section{Conclusion}

From the above result and discussion it is concluded that the ability of RANKPEP to predict MHC binding peptides, and thereby potential T-cell epitopes, antigenic peptide that binds to MHC molecule are antigenic that means hydrophilic in nature. This means the increase in affinity of MHC binding peptides may result in enhancement of immunogenicity of Dracunculus medinensis antigen Cytochrome b and are helpful in the designing of synthetic peptide vaccine. This approach can help reduce the time and cost of experimentation for determining functional properties of Dracunculus medinensis antigen Cytochrome b. Overall, the results are encouraging, both the 'sites of action' and 'physiological functions' can be predicted with very high accuracies helping minimize the number of validation experiments.

\section{References}

1. Greenaway (2004) C. Dracunculiasis (Guinea worm disease). CMAJ 170: 495500 .

2. Molyneux DH, Hopkins DR, Zagaria N (2004) Disease eradication, elimination and control: the need for accurate and consistent usage. Trends Parasitol 20 : 347-351.

3. Muller R (1971) Dracunculus and dracunculiasis. Adv Parasitol 9: 73-151.

4. Muller R (1979) Guinea worm disease: epidemiology, control, and treatment. Bull World Health Organ 57: 683-689.

5. Ruiz-Tiben E, Hopkins DR (2006) Dracunculiasis (Guinea worm disease) eradication. Adv Parasitol 61: 275-309.

6. Iriemenam NC, Oyibo WA, Fagbenro-Beyioku AF (2008) Dracunculiasis--the saddle is virtually ended. Parasitol Res 102: 343-347.

7. (2015) Parasites - Dracunculiasis (also known as Guinea Worm Disease).

8. Hopkins DR, Ruiz-Tiben E, Eberhard ML, Roy SL (2014) Progress towards global eradication of dracunculiasis- January 2013-June 2014. MMWR Morb Mortal Wkly Rep 63: 1050-1054.

9. Mbong EN, Sume GE, Danbe F, Kum WK, Mbi VO, et al. (2015) Not every worm wrapped around a stick is a guinea worm: a case of Onchocerca volvulus mimicking Dracunculus medinensis. Parasit Vectors 8: 374.

10. Richards FO, Ruiz-Tiben E, Hopkins DR (2011) Dracunculiasis eradication and the legacy of the smallpox campaign: what's new and innovative? What's old and principled? Vaccine 29 Suppl 4: D86-90.

11. Hopkins DR (2013) Disease eradication. N Engl J Med 368: 54-63.

12. Muller R (1985) Life cycle of Dracunculus medinesis. In workshop on opportunities for control of dracunculiasis: contaminated papers, Washington, DC: National Academy Press.

13. Cairncross S, Muller R, Zagaria N (2002) Dracunculiasis (Guinea worm disease) and the eradication initiative. Clin Microbiol Rev 15: 223-246.

14. Issaka-Tinorgah A, Magnussen P, Bloch P, Yakubu A (1994) Lack of effect of ivermectin on prepatent guinea-worm: a single-blind, placebo-controlled trial. Trans R Soc Trop Med Hyg 88: 346-348.

15. Bloch P, Simonsen PE, Vennervald BJ (1993) The antibody response to Dracunculus medinensis in an endemic human population of northern Ghana. J Helminthol 67: 37-48.

16. Bloch P, Simonsen PE (1998) Immunoepidemiology of Dracunculus medinensis infections II. Variation in antibody responses in relation to transmission season and patency. Am J Trop Med Hyg 59: 985-990.

17. Howell N (1989) Evolutionary conservation of protein regions in the protonmotive cytochrome $\mathrm{b}$ and their possible roles in redox catalysis. $\mathrm{J} \mathrm{Mol}$ Evol 29: 157-169.

18. Esposti MD, De Vries S, Crimi M, Ghelli A, Patarnello T, et al. (1993) Mitochondrial cytochrome b: evolution and structure of the protein. Biochim Biophys Acta 1143: 243-271.

19. Blankenship R (2009) Molecular Mechanisms of Photosynthesis. Blackwell Publishing. pp: 124-132.

20. Blakely EL, Mitchell AL, Fisher N, Meunier B, Nijtmans LG, et al. (2005) A mitochondrial cytochrome $b$ mutation causing severe respiratory chain enzyme deficiency in humans and yeast. FEBS J 272: 3583-3592.

21. Siregar JE, Syafruddin D, Matsuoka H, Kita K, Marzuki S (2008) Mutation underlying resistance of Plasmodium berghei to atovaquone in the quinone binding domain 2 (Qo(2)) of the cytochrome b gene. Parasitol Int 57: 229-232.

22. Gomase VS, Kale KV, Chikhale NJ, Changbhale SS (2007) Prediction of MHC binding peptides and epitopes from alfalfa mosaic virus. Curr Drug Discov Technol 4: 117-215.

23. Gomase VS, Kale KV (2008) In silico prediction of epitopes: a new approach for fragment based viral peptide vaccines. Int J Applied Computing 1: 39-46.

24. Gomase VS, Kale KV (2008) Approach of proteomics system architecture in plant virus's database. Int J Applied Computing 1: 33-38.

25. Gomase VS, Chitlange NR (2012) Sensitive Quantitative Predictions of MHC Binding Peptides and Fragment Based Peptide Vaccines from Taenia crassiceps. J Vaccines 3: 131. 
Citation: Mishra S, Gomase VS (2016) "Cytochrome B"- Analysis of Hydrophobicity, Surface Accessibility, Antigenicity and Prediction of MHC I and MHC II Binders from Dracunculiasis. Med chem 6: 041-046. doi:10.4172/2161-0444.1000322

26. Gomase VS, Chitlange NR (2012) Microbial Proteomics Approach for Sensitive Quantitative Predictions of MHC Binding Peptide from Taenia ovis. J Data Mining Genomics Proteomics 3: 121.

27. Sonu M, Gomase S (2015) Prediction of antigenic epitope from D. medinensis: new paradigm of synthetic vaccine development. International Conference on "Recent Research Development in Environment, Social Sciences and Humanities" (ICRRDESH-15). pp: 103-107. ISBN: 978-81-931039-8-2.

28. Sonu M, Gomase S (2015) Analysis of hydrophobicity and antigenic epitope prediction from D. Medinensis. International Conference on "Technologies for Sustainability-Engineering, information Technology, Management and the Environment" (SUSTECH-15). pp: 44-51. ISBN: 978-81-931039-7-5.

29. Sonu M, Gomase S (2015) Analysis of hydrophobicity and antigenicity of heat shock protein 70 from GWD. 2nd International Conference on "Recent Innovations in science, Engineering and Management" (ICRISEM-15). pp: 2533. ISBN: 978-81-931039-9-9.

30. Sayers EW (2012) Database resources of the National Center for Biotechnology Information. Nucleic Acids Res 40: D13-25.

31. Bairoch A, Apweiler R, Wu CH, Barker WC, Boeckmann B, et al. (2005) The Universal Protein Resource (UniProt). Nucleic Acids Res 33: D154-159.

32. Hopp TP, Woods KR (1981) Prediction of protein antigenic determinants from amino acid sequences. Proc Natl Acad Sci USA 78: 3824-3828.

33. Welling GW, Weijer WJ, van der Zee R, Welling-Wester S (1985) Prediction of sequential antigenic regions in proteins. FEBS Lett 188: 215-218.

34. Parker KC, Bednarek MA, Coligan JE (1994) Scheme for ranking potential HLA-A2 binding peptides based on independent binding of individual peptide side-chains. J Immunol 152: 163-175.

35. Larsen JE, Lund O, Nielsen M (2006) Improved method for predicting linear B-cell epitopes. Immunome Res 2: 2.

36. Kolaskar AS, Tongaonkar PC (1990) A semi-empirical method for prediction of antigenic determinants on protein antigens. FEBS Lett 276: 172-174.

37. Reche PA, Glutting JP, Reinherz EL (2002) Prediction of MHC class I binding peptides using profile motifs. Hum Immunol 63: 701-709.

38. RechePA, Reinherz EL (2003) Sequence variability analysis of human class I and class II MHC molecules: functional and structural correlates of amino acid polymorphisms. J Mol Biol 331: 623-641.
39. Craiu A, Akopian T, Goldberg A, Rock KL (1997) Two distinct proteolytic processes in the generation of a major histocompatibility complex class I presented peptide. Proc Natl Acad Sci USA 94: 10850-10855.

40. Pieters J (2000) MHC class II- restricted antigen processing and presentation. Adv Immunol 75: 159-208.

41. Bhasin M, Raghava GP (2004) Analysis and prediction of affinity of TAP binding peptides using cascade SVM. Protein Sci 13: 596-607.

42. Emini EA, Hughes JV, Perlow DS, Boger J (1985) Induction of hepatitis a virusneutralizing antibody by a virus-specific synthetic peptide. J Virol 55: 836-839.

43. Karplus PA, Schulz GE (1985) Prediction of chain flexibility in proteins: a tool for the selection of peptide antigen. Naturwissenschaften 72: 212-213.

44. Sweet RM, Eisenberg D (1983) Correlation of sequence hydrophobicities measures similarity in three-dimensional protein structure. J Mol Biol 171: 479488.

45. Kyte J, Doolittle RF (1982) A simple method for displaying the hydropathic character of a protein. J Mol Biol 157: 105-132.

46. Abraham DJ, Leo AJ (1987) Extension of the fragment method to calculate amino acid zwitterion and side chain partition coefficients. Proteins 2: 130-152.

47. Bull HB, Breese K (1974) Surface tension of amino acid solutions: a hydrophobicity scale of the amino acid residues. Arch Biochem Biophys 161: 665-670.

48. Miyazawa S, Jernigen RL (1985) Estimation of Effective Interresidue Contact Energies from Protein Crystal Structures: Quasi-Chemical Approximation. Macromolecules 18: 534-552.

49. Roseman MA (1988) Hydrophilicity of polar amino acid side-chains is markedly reduced by flanking peptide bonds. J Mol Biol 200: 513-522.

50. Wolfenden R, Andersson L, Cullis PM, Southgate CC (1981) Affinities of amino acid side chains for solvent water. Biochemistry 20: 849-855.

51. Wilson KJ, Honegger A, Stotzel RP, Hughes GJ (1981) The behaviour of peptides on reverse-phase supports during high-pressure liquid chromatography. Biochem J 199: 31-41.

52. Cowan R, Whittaker RG (1990) Hydrophobicity indices for amino acid residues as determined by high-performance liquid chromatography. Pept Res 3: 75-80.

53. Chothia $C$ (1976) The nature of the accessible and buried surfaces in proteins J Mol Biol 105: 1-12. 